\title{
O Papel do Mel no Alívio da Tosse Aguda em Crianças
}

\section{The Role of Honey in Relieving Acute Cough in Children}

Joana Ferreira de Oliveira ${ }^{1}$, Isabel Peixoto ${ }^{2}$, Elsa Rodrigues ${ }^{3}$, Laura Igreja ${ }^{4}$

\section{RESUMO}

INTRODUÇÃO: A tosse é uma queixa frequente nas consultas de Medicina Geral e Familiar. O mel tem propriedades anti-bacterianas e anti-inflamatórias, sendo usado empiricamente no tratamento da tosse. Esta revisão pretendeu avaliar o benefício do mel no alívio da tosse aguda em crianças.

METODOLOGIA: Em março/2017 foi efetuada pesquisa em nove bases de dados de ensaios clínicos aleatorizados, meta-análises, revisões sistemáticas e artigos de opinião, dos últimos 10 anos, em português e inglês. Incluíram-se estudos que avaliaram o mel no alívio da tosse aguda em crianças dos 2-14 anos de idade, comparando-o com antitússicos, placebo e ausência de tratamento. Usou-se a escala Strength of Recommendation Taxonomy para estratificar o nível de evidência (NE) e a força de recomendação (FR).

RESULTADOS: Encontraram-se 10 artigos, selecionando duas revisões sistemáticas e dois artigos de opinião. Uma revisão sistemática concluiu que o mel e o dextrometorfano parecem semelhantes no alívio da tosse e que o mel é mais eficaz que a difenidramina (NE 2). Outra revisão sistemática favoreceu o mel no alívio da tosse aguda (FR C). Um artigo de opinião referiu que o mel pode ser uma boa opção (NE 3). O artigo de opinião de Allan et al referiu pouca evidência (NE 3).

DISCUSSÃO: O mel pode ser melhor que a ausência de tratamento, difenidramina e placebo na tosse aguda, embora não pareça superior ao dextrometorfano. Por ser disponível, ter baixo preço e baixo risco, poderemos considerar o mel no tratamento da tosse aguda (SORT B) em crianças com mais de 2 anos de idade.

PALAVRAS-CHAVE: Antitússicos; Apiterapia; Criança; Tosse/tratamento 


\section{ABSTRACT}

INTRODUCTION: Cough is a frequent symptom in General Practice consultations. Honey appears to have both anti-bacterial and anti-inflammatory properties and has been used empirically in the treatment of cough. This review is intended to evaluate the benefit of honey administration in children with acute cough.

METHODS: In March/2017, randomized clinical trials, meta-analysis, systematic reviews and opinion articles of the last 10 years in Portuguese and English were searched in nine databases. Studies evaluating honey for relief of acute cough from upper respiratory infection in children 2-14 years of age were compared with antitussives, placebo, and no treatment. We used the scale Strength of Recommendation Taxonomy (SORT) to stratify the level of evidence (LOE) and the grade of recommendation (GoR).

RESULTS: We found 10 articles, two systematic reviews and two opinion articles were selected. One systematic review concluded that honey and dextromethorphan appear similar in relieving cough and that honey is more effective than diphenhydramine (LoE 2). Another systematic review favored honey in relieving acute cough (GoR C). An opinion piece mentioned that honey may be a good option (LOE 3). Allan et al article of opinion argued that there is little evidence (LOE 3).

DISCUSSION: Honey may be better than the absence of treatment, diphenhydramine and placebo in the treatment of acute cough, although it does not appear to be superior to dextromethorphan. Being available, low in price and low risk, we may consider honey in the treatment of acute cough (SORT B).

KEYWORDS: Antitussive Agents; Apitherapy; Child; Cough/therapy; Honey

\section{INTRODUÇÃO}

A tosse é um ato reflexo que resulta da estimulação dos recetores da mucosa respiratória, desde a faringe aos bronquíolos terminais. Tem várias etiologias, nomeadamente infeção ou irritação das vias aéreas, asma, alergias, presença de corpos estranhos e funciona como um importante meio de defesa da via respiratória.

É um sintoma bastante frequente nas consultas de Medicina Geral e Familiar (MGF).

Num estudo observacional e transversal, realizado no Centro de Saúde de Cascais durante 3 anos (de 1997 a 2000), onde se estudaram os motivos de consulta mais frequentes em MGF, a tosse ocupou o quinto lugar, sendo responsável por 3,2\% do total de consultas. ${ }^{1}$ Trata-se de um sintoma comum também em idade pediátrica ${ }^{2,3} \mathrm{e}$ que causa angústia nos pais e cuidadores e, por isso, motiva várias avaliações pelo Médico de Família. Tem impacto na qualidade de vida das crianças e dos seus pais, aumentando a probabilidade de absentismo de ambos e diminuindo o rendimento escolar e laboral, respetivamente. Por outro lado, está associada a maior recurso a serviços de saúde, realização de exames complementares de diagnóstico e consumo de medicamentos e outros produtos.

Muitas vezes os pais recorrem às farmácias na expectativa de diminuir o período sintomático da criança através da administração de xaropes. É importante informar os pais que no caso de aparecimento de tosse, a criança deve ser observada por um médico e que apesar da preocupação que a tosse gera, na maioria dos casos, é causada por doenças benignas e autolimitadas. Se a causa mais provável for atribuída a infeção das vias aéreas superiores, existem medidas de suporte que podem e devem ser implementadas empiricamente, nomeadamente o reforço da hidratação oral e a evicção de locais com fumo ou poeiras.

Em termos culturais, o mel é também um dos "remédios" a que se pode recorrer, tendo alguns estudos demonstrado o seu benefício no alívio sintomático. O mel parece ter propriedades anti-bacterianas ${ }^{4}$ e anti-inflamatórias, ${ }^{5}$ tendo vindo a ser usado empiricamente para o tratamento da tosse. Quando se opta pela introdução do mel, este deve ser realizado num curto período de tempo, tendo em consideração o risco de cáries dentárias e aumento do peso. Apesar de em termos culturais ser muito bem aceite o uso de mel, deve ser dada especial atenção ao facto de este poder aumentar o risco de transmissão da bactéria Clostridium botulinum e, por isso, não deve ser oferecido até aos 12 meses de idade. A Sociedade Portuguesa de Pediatria ainda não tem recomendação formal para o uso do mesmo no alívio da tosse.

Realizou-se esta revisão baseada na evidência com o intuito de perceber qual a evidência da administração de mel em crianças com tosse aguda e assim melhorar os cuidados prestados. 


\section{PERGUNTA DE REVISÃO}

Será o mel uma mais-valia no alívio sintomático das crianças com tosse aguda? utilizando-se o método Patient-Oriented Evidence that Matters (POEM).

\section{OBJETIVOS}

Esta revisão baseada na evidência teve como objetivos a avaliação dos benefícios do mel e comparação com ausência de tratamento, placebo e antitússicos em relação a:

- Frequência da tosse;

- Qualidade de vida das crianças;

- Qualidade de vida dos pais.

\section{MÉTODOS}

Foi realizada pesquisa de ensaios clínicos aleatorizados (ECA), meta-análises, revisões sistemáticas (RS) e artigos de opinião (AO), em março de 2017, publicados nos últimos 10 anos em português ou inglês em nove bases de dados médicas (National Guideline Clearinghouse; Guidelines Finder; Canadian Medical Association Practice Guidelines InfoBase; Evidence Based Medicine Online; Nice National Institute for Health and Care Excellence; Cochrane Library; University of York - DARE; Canadian Medical Association Practice Guidelines InfoBase; Pubmed). Utilizaram-se as palavras-chave (termos MeSH) Honey, Child, Child preschool, Antitussive agents e Cough.

Incluíram-se os estudos que avaliaram a eficácia do mel e o compararam com agentes antitússicos, placebo e ausência de tratamento no alívio da tosse aguda por infeção respiratória superior, em crianças dos 2 aos 14 anos de idade. A eficácia do mel foi definida pela melhoria da qualidade do sono na criança e dos pais e pela diminuição do impacto da tosse no dia-a-dia da criança.

Foram excluídos os artigos que se referiam a tosse aguda causada por patologia de base, não causada por infeção do trato respiratório superior, toma simultânea de mel com outras substâncias e toma de outras substâncias além de mel, antitússicos ou placebo.

Para estratificar o nível de evidência e a força de recomendação utilizou-se a escala Strength of Recommendation Taxonomy (SORT) da American Family Physician.

\section{RESULTADOS}

No total foram encontrados 10 artigos, dos quais quatro foram incluídos: duas revisões sistemáticas e dois artigos de opinião.

A revisão sistemática de Oduwole et al 2014, que in- cluiu três ensaios clínicos randomizados e controlados, com número total de 590 crianças, concluiu que o mel e o dextrometorfano parecem ter o mesmo efeito no alívio da tosse e, para além disto, o mel apresentava maior eficácia que a difenidramina, tendo os autores atribuído um NE 2 a este artigo.

A revisão sistemática de Ran D. Goldman (Tabela 1) foca a Norma de Orientação Clínica do Department of Child and Adolescent Health (2001) que refere que o mel pode ter um papel benéfico no tratamento da tosse em crianças e que deve ser encorajado o seu uso. No entanto, realça que são necessários mais ensaios clínicos, pelo que é atribuída uma força de recomendação C.

O artigo de opinião de Evans et al, 2010, concluiu que o mel parece ser uma opção razoável dado o seu baixo custo, bom perfil de segurança e os potenciais efeitos benéficos, sendo necessários mais ensaios clínicos randomizados para se poder recomendar o mel como aliviador da tosse. Foi atribuído um NE 3 a este artigo.

O artigo de opinião Michael Allan et al 2011, defende que a evidência da eficácia do mel na tosse aguda pediátrica é baixa, a sua significância era incerta e apresentava alto risco de viés. Foi atribuído um NE 3 a este artigo.

\section{DISCUSSÃO/CONCLUSÃO}

Apesar do mel ser uma substância há muito conhecida e utilizada empiricamente para os mais diversos fins terapêuticos, existem ainda poucos estudos que avaliem a sua eficácia no alívio de sintomas relacionados com infeções respiratórias superiores.

A toma de mel demonstrou ser eficaz na diminuição da frequência da tosse, melhoria da qualidade do sono dos pais e das crianças. Os estudos analisados nesta revisão apontam que a utilização do mel parece ter uma eficácia superior à ação da difenidramina, embora pareça ter um benefício semelhante ao dextrometorfano. Os resultados obtidos apresentam evidência em relação à eficácia do mel na melhoria da tosse e da qualidade do sono das crianças (com idade superior a 2 anos) e dos pais, demonstrando ser superior ao placebo e à ausência de tratamento e, ainda, comparável a alguns antitússicos.

Contudo, os estudos analisados incluem poucos participantes e apresentam algumas limitações metodológicas, o que não permite extrapolar os resultados para a população geral (SORT B).

Dada a sua fácil disponibilidade, preço reduzido, baixo risco de efeitos adversos e complicações poderemos considerar o mel como uma opção segura e benéfica no 
TABELA 1. Revisões sistemáticas.

Revisões sistemáticas

\begin{tabular}{|l|l|c|c|c|c|}
\hline Estudos & População & $\begin{array}{c}\text { Intervenção } \\
\text { e comparação }(\mathrm{n})\end{array}$ & Resultado & NE \\
\hline
\end{tabular}
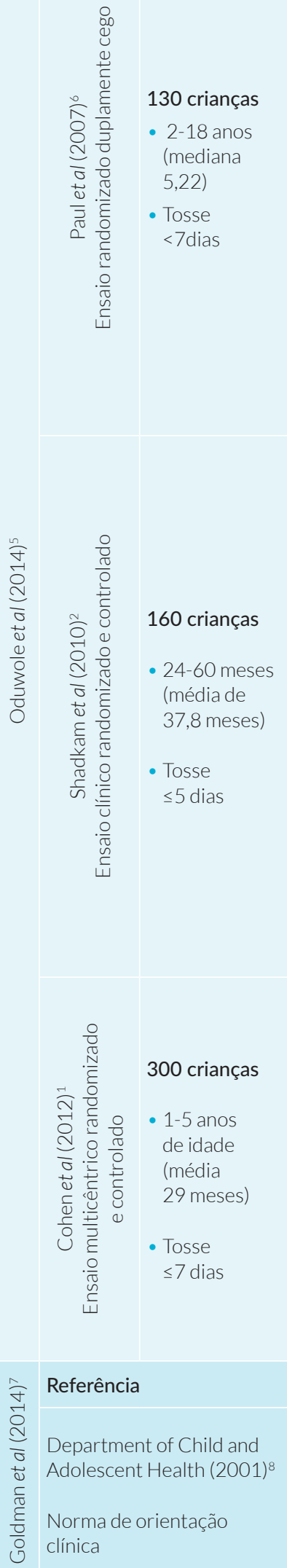

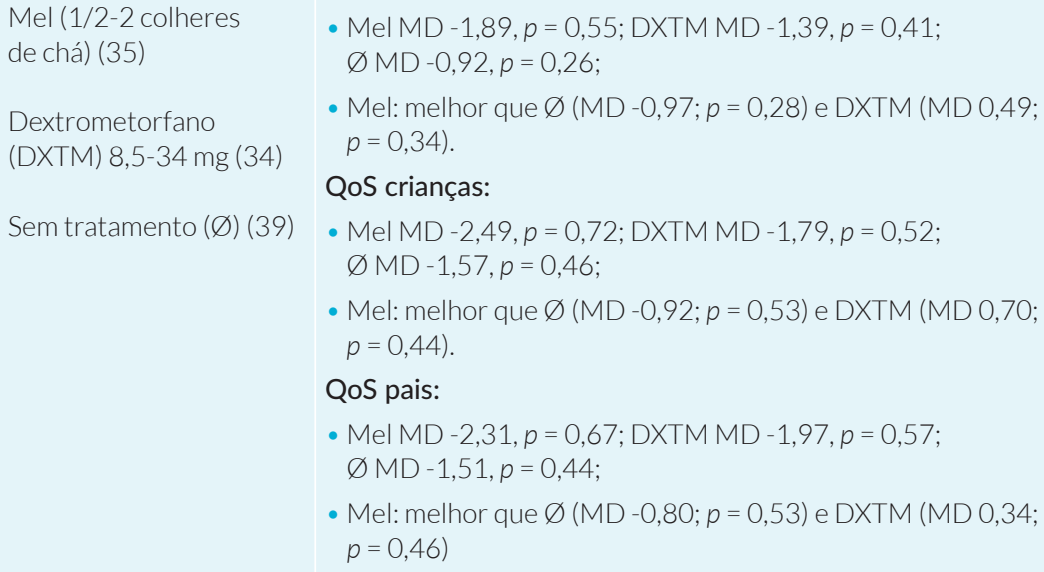

- Mel MD -1,89, $p=0,55$; DXTM MD -1,39, $p=0,41$; $\varnothing \mathrm{MD}-0,92, p=0,26$;

- Mel: melhor que $\varnothing(M D-0,97 ; p=0,28)$ e DXTM (MD 0,49; $p=0,34)$.

\section{QoS crianças:}

- Mel MD -2,49, $p=0,72 ;$ DXTM MD -1,79, $p$ = 0,52;

$\varnothing \mathrm{MD}-1,57, p=0,46$

- Mel: melhor que $\varnothing$ (MD -0,92; $p=0,53$ ) e DXTM (MD 0,70; $p=0,44)$.

QoS pais:

- Mel MD -2,31, $p=0,67$; DXTM MD -1,97, $p=0,57$; $\varnothing \mathrm{MD}-1,51, p=0,44$

- Mel: melhor que $\varnothing$ (MD -0,80; $p=0,53$ ) e DXTM (MD 0,34; $p=0,46)$

Melhoria da frequência da tosse, QoS crianças e pais em todos os grupos.

\section{Frequência da tosse:}

Melhoria estatisticamente significativa da frequência da tosse, QoS crianças e pais em todos os grupos.

\section{Frequência da tosse:}

- Mel MD -2,17, $p<0,001$; DXTM MD -1,61, $p<0,001$; DFN MD 1,73, $p<0,001 ; \varnothing \mathrm{MD}-1,08, p<0,001$

- Mel: melhor que $\varnothing$ (MD -1,18; $p<0,001)$, DXTM (MD -0,54; $p=0,001)$ e DFN (MD -0,57; $p=0,001)$

\section{QoS crianças:}

- Mel MD -2,24; $p<0,001$; DXTM MD -1,72, $p<0,001$; DFN MD-1,64, $p<0,001 ; \varnothing \mathrm{MD}-1,13, p<0,001$

- Mel: melhor que $\varnothing$ (MD -1,07; $p<0,001)$, DXTM (MD -0,49; $p=0,002)$ e DFN (MD -0,55, $p=0,001)$

\section{QoS pais:}

- Mel MD -2,39; $p<0,001$; DXTM MD -1,97, $p<0,001$; DFN MD - 1,89, $p<0,001 ; \varnothing \mathrm{MD}-1,42, p<0,001$;

- Mel: melhor que DFN (MD -0,48; $p=0,01)$, DXTM (MD -0,4; $p=0,005)$ e $\varnothing(M D-0,99 ; p<0,001)$.

Melhoria da frequência da tosse, QoS crianças e pais em todos os grupos.

\section{Frequência da tosse:}

- Mel MD -1,84, $p=0,77$; placebo MD -1,00; $p=0,42$

- Mel: melhor que placebo (MD -1,85, $p=0,77$ )

\section{QoS crianças:}

- Mel MD -1,94, $p$ = 1,02; placebo MD -1,21, $p=0,64$

- Mel: melhor que placebo (MD -1,94, $p=1,02)$

\section{QoS pais:}

- Mel MD -2,05, $p=1,11$; placebo MD -1,28, $p=0,69$

- Mel: melhor que placebo (MD -2,05, $p=1,11)$ 
TABELA 2. Artigos de opinião.

\section{Artigos de opinião}

Referência

Opinião

NE

Evans et al $(2010)^{9}$

Allan et al $(2011)^{10}$ randomizados para se poder recomendar o mel como alívio da tosse.

Há pouca evidência do benefício do mel na tosse aguda.
9. Evans $\mathrm{H}$, Tuleu $\mathrm{C}$, Sutcliffe A. Is honey a well-evidenced alternative to over-the-counter cough medicines? J R Soc Med. 2010;103:164-5. doi: 10.1258/jrsm.2010.090445.

10. Allan GM, Korownyk C, Kolber M. Do cough suppressants or honey help pediatric cough? Can Fam Physician. 2011;57:435.

tratamento da tosse aguda. No futuro são necessários mais estudos, bem desenhados, para avaliar o efeito do mel na duração da tosse.

CONFLITOS DE INTERESSE: Os autores declaram não ter qualquer conflito de interesse na realização do presente trabalho.

FONTES DE FINANCIAMENTO: Não houve qualquer fonte de financiamento na realização do presente trabalho.

CONFLICTS OF INTEREST: The authors declare that they have no conflicts of interest.

FINANCIAL SUPPORT: This work has not received any contribution, grant or scholarship.

\section{REFERÊNCIAS}

1. Cohen HA, Rozen J, Kristal H, Laks Y, Berkovitch M, Uziel Y, et al. Effect of honey on nocturnal cough and sleep quality: a double-blind, randomized, placebo-controlled study. Pediatrics. 2012;130:465-71. doi: 10.1542/peds.2011-3075.

2. Shadkam M, Mozaffari-Khosravi H, Mozayan M. A Comparison of the effect of honey, dextromethorphan, and diphenhydramine on nightly cough and sleep quality in children and their parents. J Altern Complement Med. 2010;16:787-93. doi: 10.1089/acm.2009.0311.

3. Lusby PE, Coombes AL, Wilkinson JM. Bactericidal activity of different honeys against pathogenic bacteria. Arch Med Res. 2005t;36:464-7.

4. Mullai V, Menon T. Bactericidal activity of diferent types of honey against clinical and environmental isolates of Pseudomonas aeruginosa. J Altern Complement Med. 2007;13:439-41.

5. Oduwole O, Udoh EE, Oyo-Ita A, Meremikwu MM. Honey for acute cough in children. Cochrane Database Syst Rev 2018;4:CD007094. doi: 10.1002/14651858.CD007094. pub5.

6. Paul IM, Beiler J, McMonagle A, Shaffer ML, Duda L, Berlin CM. Effect of honey, dextromethorphan, and no treatment on nocturnal cough and sleep quality for coughing children and their parents. Arch Pediatr Adolesc Med. 2007;161:1140-6.

7. Goldman RD. Honey for treatment of cough in children. Can Fam Physician. 2014 Dec;60(12):1107-8, 1110.

8. Department of Child and Adolescent Health. Cough and cold remedies for the treatment of acute respiratory infections in young children. Geneva: World Health Organization; 2001. [accessed Nov 2018] Available from: http://whqlibdoc.who. int/hq/2001/WHO_FCH_CAH_01.02.pdf. Acedido em 12/ abril/2017. 